\title{
Identification of Mycobacterium paratuberculosis gene expression signals
}

\author{
John P. Bannantine, ${ }^{1} \dagger$ Raúl G. Barletta, ${ }^{2}$ Charles O. Thoen ${ }^{1}$ \\ and Robert E. Andrews, Jr ${ }^{1}$
}

Author for correspondence: Robert E. Andrews, Jr. Tel: +1 515294 8988. Fax: +1 5152946019 . e-mail: randrews@iastate.edu

\footnotetext{
1 Department of Microbiology, Immunology and Preventive Medicine, lowa State University, Ames, IA 50011, USA

2 Department of Veterinary and Biomedical Sciences, Center for Biotechnology, University of Nebraska, Lincoln, NE 68583-0905 USA
}

\begin{abstract}
Mycobacterium paratuberculosis promoter-containing clones were isolated from a genomic DNA library constructed in the transcriptional-translational fusion vector pYUB76. The promoter-containing DNA fragments were identified in the surrogate host Mycobacterium smegmatis by expression of the promoterless lacZ reporter gene of pYUB76. The expression signals exhibited a wide range of strengths, as indicated by their corresponding $\beta$-galactosidase activities. Eight clones were sequenced and characterized further. Predicted open reading frames and codon usage were identified by computer analysis. Database searching for related sequences using the BLAST method revealed no homologies. Transcriptional activity was measured by slot-blot hybridization with steady-state RNA isolated from lacZ+ $M$. smegmatis clones. Primer extension analysis identified the transcription start sites within the cloned fragments. The promoter regions characterized in this study were used to establish a consensus promoter sequence for $\boldsymbol{M}$. paratuberculosis. $\boldsymbol{M}$. paratuberculosis consensus hexanucleotide sequences of TGMCGT and CGGCCS centred approximately 35 and 10 bp upstream from the transcription startpoints do not correspond to the consensus hexanucleotides of Escherichia coli promoters.
\end{abstract}

Keywords: Mycobacterium, promoter, gene expression, codon usage, primer extension

\section{INTRODUCTION}

Despite the medical importance of members in the genus Mycobacterium, characterization of mycobacterial gene expression signals has lagged behind other bacterial systems. Genetic manipulation is difficult because pathogenic mycobacteria are slow growing, have undeveloped methods of genetic exchange, and are refractile to chemical or enzymic lysis. For these reasons, Mycobacterium paratuberculosis ( $M$. avium subsp. paratuberculosis), the aetiological agent of Johne's disease, has not been well characterized at the molecular level. Few genes have been cloned and sequenced (Stevenson et al., 1991; Gilot et al., 1993; Green et al., 1989; Hance et al., 1989; El-Zaatari et al., 1994) and only two promoters have been characterized in this species (Murray et al., 1992; Thomas et al., 1992). No composite class trans-

\footnotetext{
†Present address: Laboratory of Intracellular Parasites, Rocky Mountain Laboratories, NIH, NIAID 903 South 4th Street, Hamilton, MT 59840, USA.

The Genome Sequence Database accession numbers for the $M$. paratuberculosis sequences determined in this work are L76360, L76361, L76363, L76364, L76365, L76373, L76374 and L76378.
}

posons have been discovered and a method of genetic exchange has only recently been established (FoleyThomas et al., 1995).

Recently, several mycobacterial promoters have been cloned (Sela \& Clark-Curtiss, 1991; Barletta et al., 1992; DasGupta et al., 1993; Thomas et al., 1992; Timm et al., 1994). In these studies the cloned promoter fragments were not sequenced (Barletta et al., 1992; Timm et al., 1994) and transcription start site mapping was either performed in recombinant Escherichia coli clones (Sela \& Clark-Curtiss, 1991) or not at all (Barletta et al., 1992; DasGupta et al., 1993). Very recently, a thorough analysis of Mycobacterium smegmatis and M. tuberculosis promoter elements has been performed (Bashyam et al., 1996).

The transcriptional-translational fusion vector pYUB76 has been used to clone expression signals from mycobacteriophages (Barletta et al., 1992). We used this vector to extend the current knowledge of promoter architecture and gene expression in $M$. paratuberculosis by cloning and characterizing chromosomal promoter elements. 


\section{METHODS}

Bacterial strains and plasmids. $M$. paratuberculosis ATCC 19698 was provided by D. Whipple, NADC, Ames, IA, USA. Escherichia coli DH10B (Gibco-BRL) served as the strain for routine plasmid maintenance and isolation. $M$. smegmatis $\mathrm{mc}^{2} 155$ (Snapper et al., 1990) was the surrogate host for the $M$. paratuberculosis gene expression experiments. The transcriptional-translational lacZ fusion vector pYUB76 (Barletta et al., 1992) replicates in E. coli and M. smegmatis.

Growth conditions and extraction of $\boldsymbol{M}$. paratuberculosis DNA. For genomic DNA isolation, $M$. paratuberculosis was grown in Middlebrook $7 \mathrm{H} 9$ broth (Difco) containing $0.5 \%$ Tween-80, OADC enrichment (Difco) and $2 \mathrm{mg} \mathrm{l}^{-1}$ mycobactin J (Allied Monitor). The cells were harvested after 5 weeks of growth in tissue-culture flasks (Corning) at $37^{\circ} \mathrm{C}$. M. paratuberculosis pellets $(1-3 \mathrm{~g})$ were frozen at $-70^{\circ} \mathrm{C}$ and genomic DNA was extracted by the method of Whipple et al. (1987).

DNA cloning. Genomic DNA from $M$. paratuberculosis was partially digested with Sau3AI and size-fractionated by agarose gel electrophoresis. Fragments in the $0 \cdot 2-2 \mathrm{~kb}$ size range were excised from the gel and purified by electroelution (Sambrook et al., 1989). Approximately $0.5 \mu \mathrm{g}$ purified genomic DNA fragments was ligated to $1 \mu \mathrm{g}$ dephosphorylated, BamHI-cleaved pYUB76 in a final volume of $10 \mu \mathrm{l}$. The ligation mixture was used to transform $M$. smegmatis $\mathrm{mc}^{2} 155$ or E. coli $\mathrm{DH} 10 \mathrm{~B}$ by electroporation. E. coli transformants were selected on Luria-Bertani (LB) medium containing $50 \mu \mathrm{g}$ kanamycin $\mathrm{ml}^{-1}$ and X-Gal $\left(45 \mu \mathrm{g} \mathrm{ml}^{-1}\right)$. M. smegmatis electrotransformants were selected on Middlebrook $7 \mathrm{H} 10$ agar (Difco) with $50 \mu \mathrm{g}$ kanamycin $\mathrm{ml}^{-1}$ and X-Gal. Blue colonies on this medium were considered candidate $M$. paratuberculosis promoter sequences.

Quantitative $\boldsymbol{\beta}$-galactosidase assays. $\beta$-Galactosidase assays for M. smegmatis were performed as described by Barletta et al. (1992). For all experiments, $\beta$-galactosidase activity is expressed in Miller units (Miller, 1972) and normalized by dividing by the $\mathrm{OD}_{600}$ readings of the respective cultures, which ranged from $0 \cdot 2$ to $0 \cdot 3$.

RNA extraction. Total cell RNA was extracted from $M$. smegmatis using the protocol described by Bashyam \& Tyagi (1994).

DNA sequencing. Sequencing reactions were performed manually with the Sequenase version 2.0 sequencing kit (United States Biochemical) for generating size markers to resolve the length of the primer extension products. The $\operatorname{lac}^{+} M$. paratuberculosis inserts in pYUB76 were sequenced with the Taq DyeDeoxy Terminator Cycle Sequencing Kit (Perkin Elmer/Applied Biosystems Division: PE/ABD). Each sequencing reaction included $100 \mathrm{ng}$ template DNA and $10 \mathrm{pmol}$ sequencing primer in a total volume of $20 \mu \mathrm{l}$. Each reaction underwent 25 cycles $\left(98^{\circ} \mathrm{C}\right.$ for $15 \mathrm{~s}, 50^{\circ} \mathrm{C}$ for $2 \mathrm{~s}, 60^{\circ} \mathrm{C}$ for $4 \mathrm{~min}$ ) in the GeneAmp PCR System 9600 thermal cycler (Perkin-Elmer). The sequencing products were purified through Centri-Sep columns (Princeton Separations) and were loaded onto $6 \%$ denaturing polyacrylamide gels in an automated sequencer (373A DNA Sequencer; PE/ABD). Template DNA for automated sequencing was prepared by one of two methods: PEG preparation (Tartof \& Hobbs, 1987) or Qiagen miniprep extraction.

To sequence $\mathrm{lacZ}^{+}$inserts from pYUB76, synthetic oligonucleotides $\left(5^{\prime}\right.$-CATTATTATCATGACATTAACC-3' and $5^{\prime}$-GTGCTGCAAGGCGATTAAGTTG-3') that flank the cloning site and hybridize to the vector were employed. When needed, internal primers were designed to sequence further into the cloned inserts.

Computer analysis of DNA sequences. Sequence alignments were performed with the software package SeqEd (Version 1.0.3; PE/ABD). Basic Local Alignment Search Tool (BLAST; Altschul et al., 1990) was used for database homology searches. The ORFs were identified with either Gene Finder, Sequencher 3.0, or the Wisconsin package GCG (Devereux et al., 1984).

Slot-blot hybridization analysis. Aliquots $(5 \mu \mathrm{g})$ of $M$. smegmatis total cell RNA were heated and chemically denatured prior to application to a Hybond- $\mathrm{N}$ nylon membrane (Amersham). RNA samples were applied with a filtration manifold consisting of a lucite block containing a number of slots (slot-blotting apparatus; Gibco-BRL). Prehybridization, hybridization and washes were performed by routine methods (Sambrook et al., 1989). The radioactive probes used for hybridization consisted of $\left[\alpha^{32} \mathrm{P}\right] \mathrm{dATP}-$ labelled $3 \cdot 2 \mathrm{~kb}$ EcoRI fragment from pCClac5 (Cupples \& Miller, 1988) and 16S and 23S rRNA from M. smegmatis. Plasmid pCClac5 contains the $3.2 \mathrm{~kb}$ EcoRI fragment carrying lacZ. Unincorporated nucleotides were separated from labelled probe by two consecutive precipitations with ethanol in the presence of $7.5 \mathrm{M}$ ammonium acetate. Slot-blot signals were quantified by scanning the autoradiogram into the shareware computer program NIH Image and performing an internal calibration. The levels of RNA were adjusted by first normalizing the relative amounts of RNA used in the rRNA slots then similarly adjusting the RNA levels in the lac $Z$ slots.

Primer extension analysis. Total cell RNA from $M$. smegmatis strains harbouring the lac $Z$ fusions was used as a template for the synthesis of cDNA products from $5^{\prime}$-end-labelled synthetic oligonucleotide primers. The primer (5'-AGTCACGACGTTGTAAAACGACG-3'), which corresponds to nucleotides internal to lacZ in pYUB76, was end-labelled by T4 polynucleotide kinase (Promega) with $\left[\gamma^{32} \mathrm{P}\right]$ ATP (Amersham). The annealing and reverse transcription reactions were performed with the AMV reverse transcriptase primer extension system following the instructions of the manufacturer (Promega). The products of the reactions were visualized by autoradiography after separation in $6 \%$ urea-polyacrylamide gels.

\section{RESULTS}

\section{Cloning $M$. paratuberculosis promoters in pYUB76}

To define $M$. paratuberculosis promoter elements, a genomic DNA library was constructed in the transcriptional-translational fusion vector pYUB76 (Barletta et al., 1992) and transformed into E. coli DH10B. This library was pooled, amplified and transformed by electroporation into $M$. smegmatis. In a representative experiment, $24 \mathrm{lacZ}^{+}$colonies were obtained from 1774 kanamycin-resistant transformants. Eight clones displaying varying intensities in the chromogenic assay (e.g. dark to light blue on X-Gal plates) were selected for further study.

\section{Relative $\beta$-galactosidase activity and mRNA levels of the $M$. smegmatis lacZ-fusion constructs}

Fig. 1 shows quantitative $\beta$-galactosidase activities and steady-state mRNA levels of $M$. smegmatis transformants harbouring the pYUB76 vector with and 


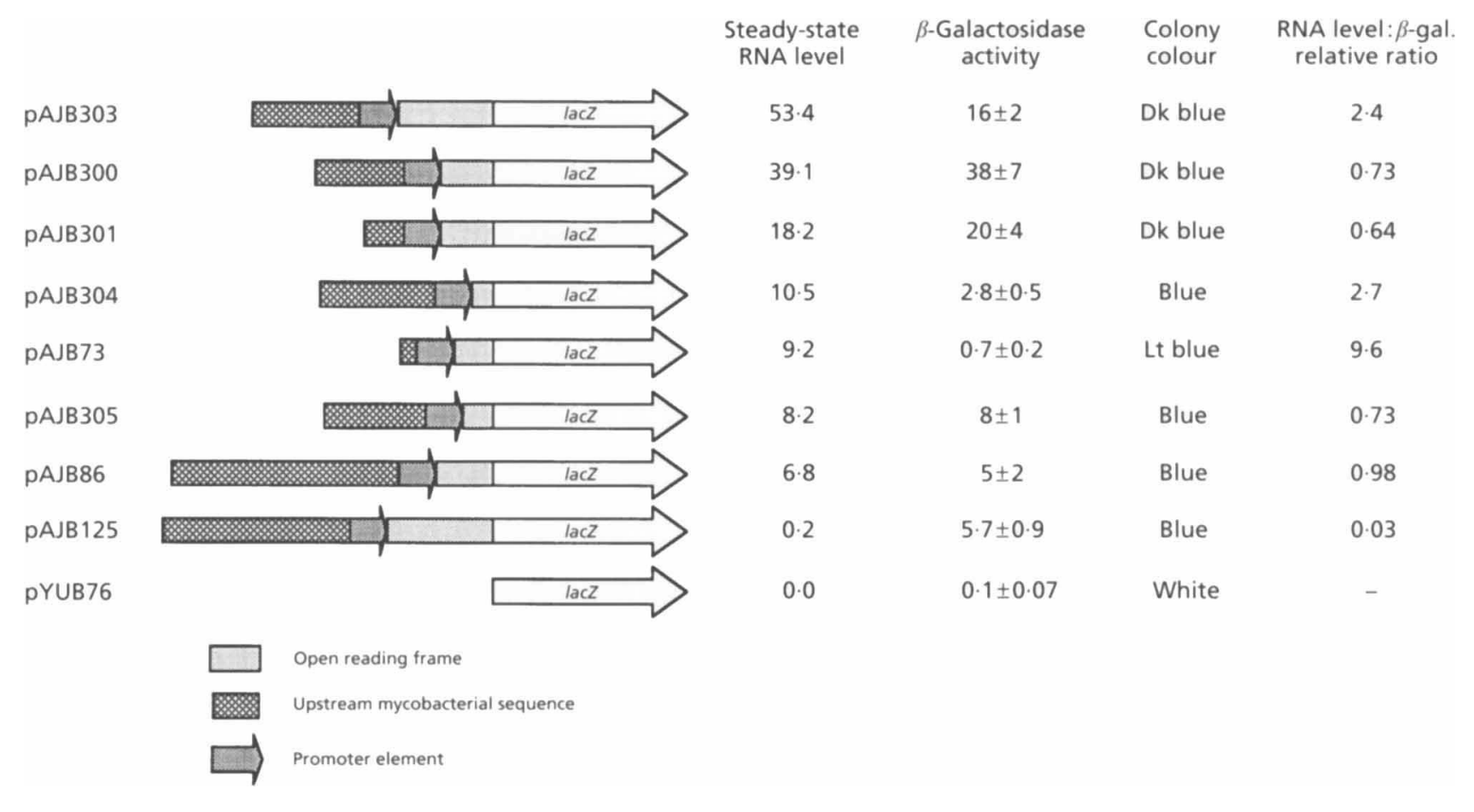

Fig. 1. Schematic diagram of the pYUB76::lacZ fusions with corresponding mRNA levels and $\beta$-galactosidase activities. The construct maps are drawn to show relative sizes of the ORFs and the upstream sequences. The transcriptional (mRNA levels, percentage density of lac $Z$ message) and translational ( $\beta$-galactosidase activities in Miller units) efficiencies are shown. The constructs are listed from highest to lowest amount of lacZ mRNA in Fig. 2 . Also indicated are the normalized ratio of mRNA levels and $\beta$-galactosidase activities [lac $Z$ mRNA/maximum lacZ mRNA (pAJB303)]/[ $\beta$-galactosidase activity/maximum $\beta$-galactosidase activity (pAJB300)].

without promoter-containing inserts. Also shown are the schematic maps of the lac $Z$ fusions in this study. The lacZ fusions pAJB300, pAJB301 and pAJB303 displayed high expression of the reporter gene; fusions $\mathrm{pAJB} 86$, $\mathrm{pAJB} 125$, and $\mathrm{pAJB} 305$ gave medium levels of $\beta$ galactosidase activity; and fusions pAJB73 and pAJB304 showed low levels. No detectable activity was observed with the strain carrying the vector pYUB76. The $M$. smegmatis (pAJB73) lacZ fusion exhibited a blue colour on X-Gal-containing media, but had little $\beta$ galactosidase activity when quantitatively determined using ONPG as the substrate. When these constructs were transformed into E. coli $\mathrm{DH} 10 \mathrm{~B}$, only pAJB73, pAJB86 and pAJB125 expressed $\beta$-galactosidase, at moderate levels (data not shown).

To quantify transcriptional activity from the pYUB76: lacZ fusions, slot-blot hybridization experiments were performed. Steady-state RNA from lacZpositive $M$. smegmatis strains was applied to nylon membranes and hybridized (Fig. 2). Comparing the relative amounts of $l a c Z$ fusion message, $M$. smegmatis cells harbouring pAJB300, pAJB301, pAJB303 and pAJB304 showed the highest level of transcriptional activity. No transcriptional activity was detected in $M$. smegmatis harbouring pYUB76 by either Northern hybridization analysis (data not shown) or slot-blot analysis (Fig. 2). As indicated by the normalized ratio of mRNA levels and $\beta$-galactosidase activities, the cor-

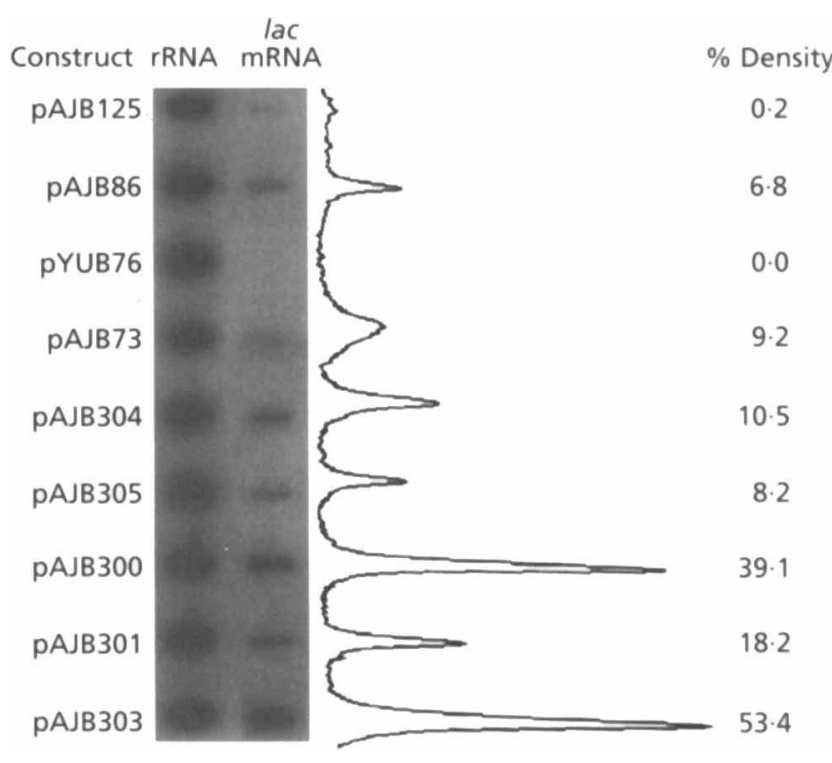

Fig. 2. Quantification of steady-state RNA from $M$. smegmatis harbouring pYUB76::lacZ fusions. Total cell RNA from $M$. smegmatis was quantitatively spotted onto a nylon membrane and hybridized with either radiolabelled rRNA from $M$. smegmatis (left column) or a lacZ-specific fragment from pCClac5 (right column). The strength of the signals was determined using an internal calibration in the computer program $\mathrm{NIH}$ Image. The results are reported as \% density: (intensity of lac $Z$ mRNA/intensity of rRNA) $\times 100$. 
Table 1. Sequence data from lac $Z^{+}$fusion constructs

\begin{tabular}{|lccc|}
\hline Construct & $\begin{array}{c}\text { Insert } \\
\text { size }(\mathrm{bp})\end{array}$ & $\begin{array}{c}\mathrm{G}+\mathrm{C} \text { content } \\
(\mathbf{m o l} \%)\end{array}$ & $\begin{array}{c}\text { No. of aa derived from } \\
\text { the mycobacterial gene }\end{array}$ \\
\hline pAJB303 & 610 & $67 \cdot 2$ & 95 \\
pAJB300 & 412 & $66 \cdot 1$ & 24 \\
pAJB301 & 292 & $70 \cdot 9$ & 61 \\
pAJB304 & 663 & $68 \cdot 2$ & 7 \\
pAJB73 & 104 & $69 \cdot 2$ & 15 \\
pAJB305 & 559 & $71 \cdot 0$ & 10 \\
pAJB86 & $>1800$ & $66 \cdot 3$ & 20 \\
pAJB125 & 1660 & $60 \cdot 8$ & 81 \\
\hline
\end{tabular}

relation between these two measurements is not complete. The pAJB303 construct shows the most steadystate mRNA, but $\beta$-galactosidase activity ranks it third strongest. Similarly, pAJB300 has the highest $\beta$-galactosidase activity and is second to pA JB303 in transcriptional activity. pAJB125 shows the least correlation, displaying medium levels of $\beta$-galactosidase activity, but very low lacZ mRNA levels. The reverse situation occurs with pAJB73. This lack of correlation is probably attributable to several factors, such as mRNA stability and translational efficiency.

\section{DNA sequence analysis}

The nucleotide sequence was determined for each of the $\mathrm{lacZ}^{+}$M. paratuberculosis inserts. Insert sizes, $\mathrm{mol} \%$ $\mathrm{G}+\mathrm{C}$, and number of amino acids derived from each mycobacterial fusion, are listed in Table 1 . The mol \% $\mathrm{G}+\mathrm{C}$ of sequenced inserts agrees well with the published figure of $66-67 \mathrm{~mol} \%$ for M. paratuberculosis DNA (McFadden et al., 1987). The putative ORFs were identified by computer analysis and confirmed for conditions specific to lac $Z$ expression in pYUB76 [i.e. all cloned sequences must contain a promoter, ribosomebinding site (RBS), and a start codon that must be inframe with the ninth codon (GUC) of lacZ]. The pAJB303 construct has two possible in-frame start codons (AUG and GUG). Since the GUG codon is separated from the RBS by only two nucleotides, we chose the AUG start codon which is separated by 8 nucleotides, a more prototypical spacing seen in prokaryotes. BLAST searches of nucleic acid databases showed no significant homology to any of the $M$. paratuberculosis sequences in this study. However, pAJB73 and pAJB304 have a $M$. paratuberculosis nucleotide or protein sequence of insufficient length to reveal meaningful homologies in the database.

\section{Primer extension analysis}

To localize the promoter region within the cloned $M$. paratuberculosis inserts, the transcription start sites were mapped. The mRNA initiation sites of the cloned lacZ-fusion transcripts were determined by primer

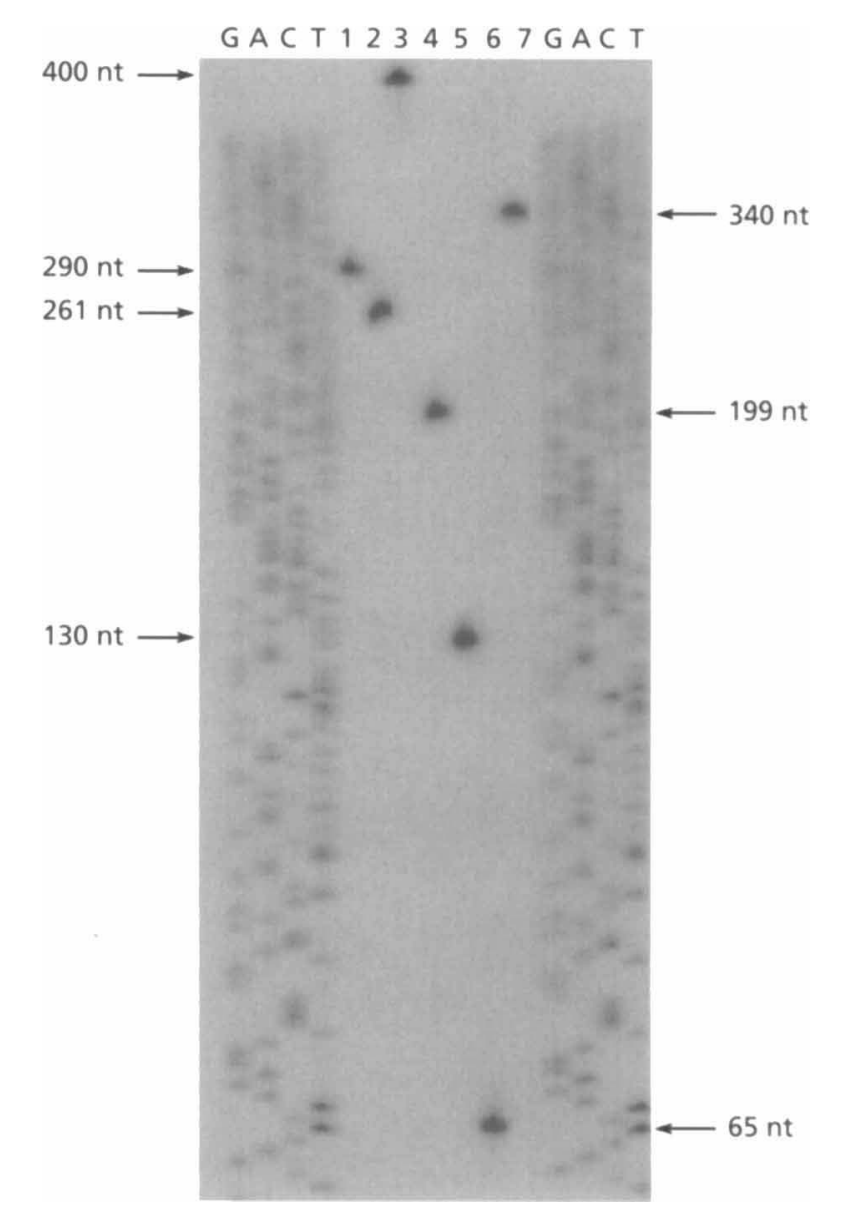

Fig. 3. Primer extension analysis of lac $Z$ fusion transcripts from pAJB300 (lane 1), pAJB301 (lane 2), pAJB303 (lane 3), pAJB86 (lane 4), pAJB305 (lane 5), pAJB125 (lane 6), and pAJB304 (lane 7). The positions of the primer extension products are indicated by arrows along with the nucleotide (nt) length of the product. An M13 sequence ladder was used to size the extension products with the potential limitation of a 1-2 nucleotide inaccuracy. Details of the experiment are described in Methods.

extension analysis. Primer extension products were generated for the pAJB73 (data not shown), pAJB86, pAJB125, pAJB300, pAJB301, pAJB303, pAJB304 and 
Table 2. Nucleotide sequences of predicted $M$. paratuberculosis ribosome-binding sites

Predicted RBSs are underlined, with nucleotides that may base-pair with rRNA shown in bold. Potential AUG and GUG initiation codons are also underlined.

\begin{tabular}{|c|c|c|c|}
\hline Construct & Nucleotide sequence & $\begin{array}{c}\Delta G \\
\left(\mathrm{kcal} \mathrm{mol}^{-1}\right)\end{array}$ & $\begin{array}{l}\text { Position of } \\
\text { start codon }\end{array}$ \\
\hline pAJB303 & UGGGCUGGGCGGGCUUGCGGAAUCACCGAAAAGAUAAGUGAAAAUG & $-6 \cdot 0$ & +94 \\
\hline pAJB300 & GGACGAUUUCACGAACCUGUGUACGCAAGUUACAGUUAUCUCCAUG & $-4 \cdot 8$ & +96 \\
\hline pAJB301 & CGACCAGGGUUUCAUGCACGGCGCUGGCGACCAUCAGCCUGCGGUG & $-5 \cdot 6$ & +59 \\
\hline pAJB304 & GAGCUGGACGGGAGGUCGUCGAUG & -15 & +22 \\
\hline pAJB73 & UGCUCGAGCUCGAGCUCGGUGUG & -3.6 & +15 \\
\hline pAJB305 & GCGACAAGGCAAUGUUGUCGGUACCGGAUGAGAUGGUCUCUUCAUG & -10 & +79 \\
\hline pAJB86 & GGCUACCGCGUCGAAGCCGGAACCAUGACUGGCGGUUCGGCAGAAUG & $-8 \cdot 0$ & +118 \\
\hline pAJB125 & AGGAGGUCGCCGGCAUCAGCGCCGGCACCACGUUGUUGCGCCACGUG & -19 & +76 \\
\hline $16 \mathrm{~S}$ rRNA $3^{\prime}$ & AUUCCUCCA.CUA & & \\
\hline
\end{tabular}

Table 3. -35 and -10 promoter regions and their spacings in mycobacteria

\begin{tabular}{|c|c|c|c|c|c|c|c|}
\hline Construct & \multicolumn{2}{|r|}{-35 region } & \multicolumn{3}{|c|}{-10 region +1} & Spacing* & $\begin{array}{l}\text { Initial } \\
\text { codon }\end{array}$ \\
\hline \multicolumn{8}{|c|}{ M. paratuberculosis promoters from this study $\dagger$} \\
\hline pAJB303 & \multicolumn{2}{|c|}{ GGTGGCGTCGCCGGTGTAGCCGAA } & \multicolumn{2}{|c|}{ CGGCACGTGCGCG } & $\mathrm{T}$ & 92 & ATG \\
\hline pAJB300 & \multicolumn{2}{|c|}{ CCTGACGCTGGCCGACCTCGGCGCG } & \multicolumn{2}{|c|}{ CAGCCGACCGCGC } & A & 195 & ATG \\
\hline pAJB301 & \multicolumn{5}{|c|}{ 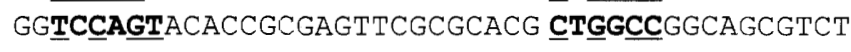 } & 55 & GTG \\
\hline pAJB304 & \multicolumn{2}{|c|}{ CCAAGGACAACGGCCGTATCCGGTA } & \multicolumn{2}{|c|}{ CCAACGGGTGTGC } & G & 21 & ATG \\
\hline pAJB73 & \multicolumn{4}{|c|}{ TGTGCCGCTTGAACCGGCCCAGCTCCCG $\underline{\mathbf{C T C C A G}}$ GGTGACG } & $\mathrm{T}$ & 13 & GTG \\
\hline pAJB305 & \multicolumn{2}{|c|}{ GTTGTTGGAGTTTCTGTCGGACGGT } & \multicolumn{2}{|c|}{ TEGTTGGCGGCAT } & $\mathrm{T}$ & 77 & ATG \\
\hline pAJB86 & \multicolumn{2}{|c|}{ GATGACGTTGCACGGCTGGGATTAA } & \multicolumn{2}{|c|}{ CGGTCCGCGTGC } & $\mathrm{T}$ & 116 & ATG \\
\hline pAJB125 & \multicolumn{2}{|c|}{ CATTAAAGATCGANGGCGCCGGGNT } & \multicolumn{2}{|c|}{ CATGTECCTTCAC } & $\mathrm{C}$ & 74 & GTG \\
\hline Consensusł & TGMCGT & $--\mathrm{N}_{16-20^{--}}$ & CGGCCS & & & & \\
\hline \multicolumn{8}{|c|}{ Other mycobacterial promoters $\S$} \\
\hline hsp60 BCG & TTGEAC & $17 *$ & TAAGAA & $6 *$ & $\mathrm{~T}$ & 183 & ATG \\
\hline $\mathrm{P}_{\mathrm{AN}} M . p t b$. & ICGACA & 17 & TACACT & 7 & A & 40 & ATG \\
\hline $16 \mathrm{~S}$ rRNA leprae & $\underline{T} T G A C T$ & 16 & ATTAAT & 7 & G & & \\
\hline $16 \mathrm{~S}$ rRNA M. $t u b$. & TTGACT & 18 & TAGACT & 7 & $\mathrm{~T}$ & & \\
\hline$c p n 60$ M. tub. & $\underline{\mathrm{TGCTCA}}$ & 17 & GGCGGC & 7 & A & 29 & ATG \\
\hline$m p b 70$ BCG & CCGATC & 17 & $\underline{\mathrm{CAT}} \underline{\mathrm{CA}} \underline{\mathrm{G}}$ & 6 & G & 176 & ATG \\
\hline bla fortuitum & TTCAAA & 19 & TACGET & 7 & $\mathrm{~A}$ & 0 & ATG \\
\hline ask smegmatis & CCㄷAG & 17 & $\mathrm{ACGCT} \underline{\mathrm{G}}$ & 7 & G & 250 & GTG \\
\hline 85A M. tub. & $\underline{T T G A C} \underline{\underline{I}}$ & 22 & $\underline{\mathrm{CGC}} \overline{\mathrm{CT}} \underline{\underline{G}}$ & 7 & A & 63 & ATG \\
\hline
\end{tabular}

* Number of nucleotides between indicated regions.

† Nucleotides in bold represent the -35 and -10 consensus regions. Underlined nucleotides match the consensus at that position. $\neq \mathrm{M}$ represents $\mathrm{C}$ or $\mathrm{A} ; \mathrm{S}$ represents $\mathrm{G}$ or $\mathrm{C}$. pAJB125 and pAJB304 were excluded when calculating the consensus.

$\$$ Mycobacterium sp. promoter regions as compiled by Kremer et al. (1995). M. ptb., M. paratuberculosis; M. tub., M. tuberculosis. Only promoters for which the transcription start sites are known were included. Underlined nucleotides indicate a match to the $M$. paratuberculosis consensus sequence.

pAJB305 constructs (Fig. 3). The startpoint of the pAJB303 transcript was not determined to single basepair accuracy due to the large size of the cDNA product. Therefore, an estimate of the extension product was made using radiolabelled $\phi$ X174 DNA markers digested with HinfI (data not shown).

\section{Putative ribosome-binding sites in $\boldsymbol{M}$. paratuberculosis mRNA}

A region of homology to the $3^{\prime}$ terminus of M. leprae $16 \mathrm{~S}$ rRNA followed after a spacing of $4-37$ bp by an initiation codon and an ORF for translation was 
observed for all the lac $Z$ fusions. Nucleotides that may base-pair with rRNA are shown in bold in Table 2 . The predicted free energy of base-pairing $(\Delta G)$ of these RBSs with $16 \mathrm{~S}$ rRNA, calculated according to the rules of Tinoco et al. (1973), ranged from -3.6 to $-19 \mathrm{kcal} \mathrm{mol}^{-1}\left(-15.5\right.$ to $\left.-79.5 \mathrm{~kJ} \mathrm{~mol}^{-1}\right)$.

\section{Codon usage}

A compilation of the codons employed in the $\mathrm{N}$-terminal mycobacterial ORFs which participated in the lac $Z$ fusions was performed (data not shown). The 309 codons compiled for $M$. paratuberculosis in this study show a characteristic GC bias, with $73 \%$ of the codons containing a $G$ or $C$ in the third position, whereas codons ending in $\mathrm{T}$ and $\mathrm{A}$ are represented at frequencies of 15 and $12 \%$, respectively. This codon usage is remarkably similar to that obtained for $M$. leprae (Honoré et al., 1993) and the M. tuberculosis complex (Andersson \& Sharp, 1996).

\section{Determination of a consensus promoter sequence}

The eight $M$. paratuberculosis promoters that had their transcription start sites identified by primer extension analysis were aligned as shown in Table 3 . The nucleotides shown in bold in Table 3 represent the putative -35 and -10 regions. Based on these data, a $M$. paratuberculosis consensus sequence for the -35 region was established as TGMCGT. No strong consensus was established for any of the positions in the -10 region. However, for the aligned sequences in Table 3, a cytosine nucleotide was present in the first position of the highlighted -10 region in $87 \%$ of cases. Additionally, the occurrence at the other positions was $37 \%$ G, $62 \%$ G, $37 \% \mathrm{C}, 50 \% \mathrm{C}$ and $100 \% \mathrm{~S}$. The putative promoter regions from pAJB125 and pAJB304 were excluded from the calculation of the consensus since those two promoter regions differed significantly from the other six. For comparison, the -35 and -10 regions of all mycobacterial promoters that contain an experimentally determined transcription start site were also compiled; these are listed at the bottom of Table 3 . Nucleotides that match the consensus are underlined. None of these promoter regions display significant homology with the M. paratuberculosis consensus.

\section{DISCUSSION}

Eight authentic $M$. paratuberculosis promoter elements were identified in this study based on transcriptional/ translational lacZ fusions and primer extension analysis using M. smegmatis as a surrogate host. Ideally, gene expression studies should be performed in the native host. However, a genetic system for M. paratuberculosis has only recently been developed (Foley-Thomas et al., 1995) and the expression of the lac $Z$ reporter gene in $M$. paratuberculosis has not been documented. Nevertheless, a homologous mycobacterial system such as $M$. smegmatis offers clear advantages to the use of an E. coli system that is unable to recognize many mycobacterial expression sequences (Barletta et al., 1992; ClarkCurtiss et al., 1985; Thole et al., 1985).

Strong $\beta$-galactosidase activity was observed in the $l a c Z$ fusion constructs of pAJB300, pAJB301 and pAJB303. The Miller units obtained in the quantitative $\beta$-galactosidase assay indicated that pAJB300 is stronger than most of the mycobacteriophage promoters cloned in pYUB76 (Barletta et al., 1992). In contrast, pAJB301 and pAJB303 were slightly weaker than the cloned mycobacteriophage promoters. The cloned pAJB300 or pAJB303 inserts may be good candidates for expression of heterologous antigens in $M$. bovis BCG for recombinant vaccine development (Stover et al., 1991, 1993) because of their high level of expression.

The slot-blot hybridization of M. smegmatis RNA was performed to quantify steady-state levels of the lac Z fusion message. Thus, pAJB303 might contain the strongest promoter. Alternatively, the pAJB303 message might be more stable. The $5^{\prime}$ leader sequence of some stable transcripts such as the T4 bacteriophage gene 32 or the E. coli ompA can stabilize other sequences fused downstream from them (Ehretsmann et al., 1992). For example, the lac $Z$ transcript has a half-life of $90 \mathrm{~s}$, but a gene 32 : : lac $Z$ fusion has a half-life of $20 \mathrm{~min}$.

Although we have not identified initiation codons directly by amino acid sequencing, all of the lac $Z$ fusion mRNAs display a putative RBS complementary to the $3^{\prime}$ region of $M$. leprae $16 \mathrm{~S}$ rRNA followed by a possible initiation codon and an open reading frame. The free energies of interaction $(\Delta G)$ for the proposed RBSs vary from -3.6 to $-19 \mathrm{kcal} \mathrm{mol}^{-1}(-15.5$ to $-79.5 \mathrm{~kJ} \mathrm{~mol}^{-1}$ ) as compared to $-9.4 \mathrm{kcal} \mathrm{mol}^{-1}$ $\left(39.3 \mathrm{~kJ} \mathrm{~mol}^{-1}\right)$ for the prototype E. coli RBS AGGA (McLaughlin et al., 1981).

Six of the promoters transcriptionally mapped in this study display some degree of conformity in their -35 regions and, less so, in the -10 regions, with the canonical sequence including matches in at least half of the positions for each region. The intervening sequence between the -35 and -10 regions ( $16-20 \mathrm{bp}$ ) corresponds to the preferred interval for prokaryotic promoters. The -35 promoter regions from pAJB304 (no position conserved) and pAJB125 (two positions conserved) differ significantly. pAJB125 also differs significantly at the -10 box (two positions conserved). Significant differences are observed when we compare the $M$. paratuberculosis consensus sequence with nine previously studied promoters (Table 3 ). These deviations suggest that these promoters may be under the control of different sigma factors, a well-known situation in other microbial systems (Hawley \& McClure, 1983). Since $M$. paratuberculosis promoters were identified in vitro under standard growing conditions, it is likely that the consensus sequence established in this study corresponds to the principal sigma factor involved in the transcription of housekeeping genes. In addition, since $M$. smegmatis was the surrogate host for these promoter studies, there may exist a bias toward $M$. paratuberculosis promoters that are highly active in that 
host. Conversely, some of the previously studied promoters may require special sigma factors related to stress conditions, as exemplified by the heat-shock promoters hsp60 and $m p b 70$.

Recently, Bashyam et al. (1996) reported a thorough study on the consensus sequences of $M$. smegmatis and $M$. tuberculosis promoters. In that study, a fairly wellconserved -10 box was found which resembled the $E$. coli Pribnow box. In contrast, we failed to find any conserved sequence of this kind. These differences may be attributable to the use of different reporter genes and selection markers, which may have led to the isolation of a different set of promoters. The observation that a bacterial sigma factor can recognize promoters with diverse -10 regions is unusual but not unique. Studies showing diverse -10 hexamers have been described for Caulobacter crescentus (Malakooti et al., 1995) and Chlamydia trachomatis (Douglas \& Hatch, 1996).

Additional promoter sequences must be compared to test and refine the consensus regions. The -35 region of the paratuberculosis consensus bears some resemblance to $E$. coli whereas the -10 region does not. Upstream regions of mycobacterial genes have been shown to have a higher $\mathrm{G}+\mathrm{C}$ content than their $E$. coli counterparts (Dale \& Patki, 1990). This observation was confirmed in the present study, as the conserved regions have a $\mathrm{G}+\mathrm{C}$ content of $66.7 \mathrm{~mol} \%$ compared with $16.7 \mathrm{~mol} \%$ for the E. coli consensus. As was pointed out by Dale \& Patki (1990), analysis of the sequence of many mycobacterial genes does not reveal any regions upstream from the putative translational start position that resemble an $E$. coli-like consensus promoter. The $M$. paratuberculosis promoters defined in this study are more closely related to each other than they are to the other compiled mycobacterial promoters in Table 3. The results of this study have, for the first time, revealed a potential paratuberculosis consensus promoter.

\section{ACKNOWLEDGEMENTS}

We thank the DNA sequencing and synthesis facility (1184 Molecular Biology Building, Iowa State University) for performing the automated sequencing of the pYUB76::lacZ fusion inserts. We also thank Greg Phillips for providing plasmids and thoughtful discussions.

This is journal paper no. J-16741 of the Iowa Agriculture and Home Economics Experiment Station, Ames, Iowa. The work is part of Project no. 3222, and was supported by Hatch Act and State of Iowa Funds. R.G.B. was supported by Cooperative State Research Service Project NEB 14-077.

\section{REFERENCES}

Altschul, S. F., Gish, W., Miller, W., Myers, E. W. \& Lipman, D. J. (1990). Basic local alignment search tool. J Mol Biol 215, 403-410.

Andersson, S. G. E. \& Sharp, P. M. (1996). Codon usage in the Mycobacterium tuberculosis complex. Microbiology 142, 915-925.

Barletta, R. G., Kim, D. D., Snapper, S. B., Bloom, B. R. \& Jacobs, W. R., Jr (1992). Identification of expression signals of the mycobacteriophages Bxb1, L1 and TM4 using the Escherichia-
Mycobacterium shuttle plasmids pYUB75 and pYUB76 designed to create translational fusions to the lacZ gene. J Gen Microbiol 138, 23-30.

Bashyam, M. D. \& Tyagi, A. K. (1994). An efficient and high yielding method for isolation of RNA from mycobacteria. BioTechniques 17, 834-836.

Bashyam, M. D., Kaushal, D., DasGupta, S. K. \& Tyagi, A. K. (1996). A study of the mycobacterial transcriptional apparatus: identification of novel features in promoter elements. J Bacteriol 178, 4847-4853.

Clark-Curtiss, J. E., Jacobs, W. R., Doherty, M. A., Ritchie, L. R. \& Curtiss, R., III (1985). Molecular analysis of DNA and construction of genomic libraries of M. leprae. J Bacteriol 161, 1093-1102.

Cupples, C. G. \& Miller, J.H. (1988). Effect of amino acid substitutions at the active site in Escherichia coli $\beta$-galactosidase. Genetics 120, 637-644.

Dale, J. W. \& Patki, A. (1990). Mycobacterial gene expression and regulation. In Molecular Biology of the Mycobacteria, pp. 173-198. Edited by J. J. McFadden. London: Surrey University Press.

DasGupta, S. K., Bashyam, M. D. \& Tyagi, A. K. (1993). Cloning and assessment of mycobacterial promoters by using a plasmid shuttle vector. J Bacteriol 175, 5186-5192.

Devereux, J., Haeberli, P. \& Smithies, O. (1984). A comprehensive set of sequence analysis programs for the VAX. Nucleic Acids Res 12, 387-395.

Douglas, A. L. \& Hatch, T.P. (1996). Mutagenesis of the P2 promoter of the major outer membrane protein gene of Chlamydia trachomatis. J Bacteriol 178, 5573-5578.

Ehretsmann, C. P., Carpousis, A. J. \& Krisch, H. M. (1992). mRNA degradation in procaryotes. FASEB (Fed Am Soc Exp Biol) J 6, 3186-3192.

El-Zaatari, F. A. K., Naser, S. A., Engstrand, L., Hachem, C. Y., Graham, D. Y. (1994). Identification and characterization of Mycobacterium paratuberculosis recombinant proteins expressed in E. coli. Curr Microbiol 29, 177-184.

Estrada-G, I. C. E., Lamb, F. I., Colston, M. J. \& Cox, R. A. (1988). Partial nucleotide sequence of $16 \mathrm{~S}$ ribosomal RNA isolated from armadillo-grown Mycobacterium leprae. J Gen Microbiol 134, 1449-1453.

Foley-Thomas, E. M., Whipple, D. L., Bermudez, L. E. \& Barletta, R. G. (1995). Phage infection, transfection, and transformation of Mycobacterium avium complex and Mycobacterium paratuberculosis. Microbiology 141, 1173-1181.

Gilot, P., De Kesel, M., Machtelinckx, L., Coene, M. \& Cocito, C. (1993). Isolation and sequencing of the gene coding for an antigenic 34-kilodalton protein of Mycobacterium paratuberculosis. J Bacteriol 175, 4930-4935.

Green, E. P., Tizard, M. L. V., Moss, M. T., Thompson, J., Winterbourne, D. J., McFadden, J. J. \& Hermon-Taylor, J. (1989). Sequence and charateristics of IS900, an insertion element identified in a human Crohn's disease isolate of Mycobacterium paratuberculosis. Nucleic Acids Res 17, 9063-9073.

Hance, A. J., Grandchamp, B., Levy-Frebault, V., Lecossier, D., Rauzier, J., Bocart, D. \& Gicquel, B. (1989). Detection and identification of mycobacteria by amplification of mycobacterial DNA. Mol Microbiol 3, 843-849.

Hawley, D. K. \& McClure, W. R. (1983). Compilation and analysis of Escherichia coli promoter DNA sequences. Nucleic Acids Res 11, 2237-2255.

Honoré, N., Bergh, S., Chanteau, S. \& 15 other authors (1993). Nucleotide sequence of the first cosmid from the Mycobacterium 
leprae genome project: structure and function of the Rif-Str regions. Mol Microbiol 7, 207-214.

Kremer, L., Baulard, A., Estaquier, J., Content, J., Capron, A. \& Locht, C. (1995). Analysis of the Mycobacterium tuberculosis $85 \mathrm{~A}$ antigen promoter region. $J$ Bacteriol 177, 642-653.

McFadden, J. J., Butcher, P. D., Chiodini, R. J. \& Hermon-Taylor, J. (1987). Determination of genome size and DNA homology between unclassified Mycobacterium species isolated from patients with Crohn's disease and other mycobacteria. J Gen Microbiol 133, 211-214.

McLaughlin, J. R., Murray, C. L. \& Rabinowitz, J. C. (1981). Unique features in the ribosome binding site sequence of the Grampositive Staphylococcus aureus $\beta$-lactamase gene. J Biol Chem 256, 11283-11291.

Malakooti, J., Wang, S. P. \& Ely, B. (1995). A consensus promoter sequence for Caulobacter crescentus genes involved in biosynthetic and housekeeping function. J Bacteriol 177, 4372-4376.

Miller, J. H. (1972). Experiments in Molecular Genetics. Cold Spring Harbor, N Y: Cold Spring Harbor Laboratory.

Moran, C. P., Jr, Lang, N., LeGrice, S. F. J., Lee, G., Stephens, M., Sonenshein, A. L., Pero, J. \& Losick, R. (1982). Nucleotide sequences that signal the initiation of transcription and translation in Bacillus subtilis. Mol Gen Genet 186, 339-346.

Murray, A., Winter, N., Lagranderie, M., Hill, D. F., Rauzier, J., Timm, J., Leclerc, C., Moriarty, K. M., Gheorghiu, M. \& Gicquel, B. (1992). Expression of Escherichia coli $\beta$-galactosidase in $\mathrm{Myco}$ bacterium bovis BCG using an expression system isolated from Mycobacterium paratuberculosis which induced humoral and cellular immune responses. Mol Microbiol 6, 3331-3342.

Muto, A. \& Osawa, S. (1987). The guanine and cytosine content of genomic DNA and bacterial evolution. Proc Natl Acad Sci USA 84, 166-169.

Sambrook, J., Fritsch, E. F. \& Maniatis, T. (1989). Molecular Cloning: a Laboratory Manual. Cold Spring Harbor, NY: Cold Spring Harbor Laboratory.

Sela, S. \& Clark-Curtiss, J. (1991). Cloning and characterization of the Mycobacterium leprae ribosomal RNA promoter in Escherichia coli. Gene 98, 123-127.

Snapper, S. B., Melton, R. E., Mustafa, S., Kieser, T. \& Jacobs, W. R. (1990). Isolation and characterization of efficient plasmid transformation mutants of Mycobacterium smegmatis. Mol Microbiol 4, 1911-1919.

Stevenson, K., Inglis, N. F., Rae, B., Donachie, W. \& Sharp, J. M. (1991). Complete nucleotide sequence of a gene encoding the $70 \mathrm{kd}$ heat shock protein of Mycobacterium paratuberculosis. Nucleic Acids Res 19, 4552.

Stover, C. K., de la Cruz, V. F., Fuerst, T. R. \& 11 other authors (1991). New use of BCG for recombinant vaccines. Nature 351, $456-460$.

Stover, K., Bansal, G. P., Hanson, M. S. \& 7 other authors (1993). Protective immunity elicited by recombinant bacille-CalmetteGuérin (BCG) expressing outer surface protein A (Osp A) lipoprotein: a candidate lyme disease vaccine. J Exp Med 178, 197-209.

Tartof, K. D. \& Hobbs, C. A. (1987). Improved media for growing plasmid and cosmid clones. Bethesda Res Lab Focus 9, 12.

Thole, J. E. R., Dauwerse, H. G., Das, P. K., Groothjuis, D. G., Schouls, L. M. \& van Embden, J. D. A. (1985). Cloning of Mycobacterium bovis BCG DNA and expression of antigens in Escherichia coli. Infect Immun 50, 800-806.

Thomas, T. J., Thoen, C. O. \& Andrews, R. E., Jr (1992). Molecular cloning and characterization of Mycobacterium paratuberculosis promoters in Escherichia coli. Vet Microbiol 32, 351-362.

Timm, J. M., Perilli, G., Uez, C. \& 9 other authors (1994). Transcription and expression analysis using $l a c Z$ and $p h o A$ gene fusions of Mycobacterium fortuitum $\beta$-lactamase genes cloned from a natural isolate and a high level $\beta$-lactamase producer. $\mathrm{Mol}$ Microbiol 12, 491-504.

Tinoco, I., Borer, P. N., Dengler, B., Levine, M. D., Uhlenbeck, O. C., Crothers, D. M. \& Gralla, J. (1973). Improved estimation of secondary structure in ribonucleic acids. Nature New Biol 246, $40-41$.

Whipple, D. L., Le Febvre, R. B., Andrews, R. E., Jr \& Thiermann, A. B. (1987). Isolation and analysis of restriction endonuclease digestive patterns of chromosomal DNA from Mycobacterium paratuberculosis and other Mycobacterium species. J Clin Microbiol 25, 1511-1515.

Received 9 August 1996; revised 25 October 1996; accepted 28 October 1996. 